\title{
Vibrionaceae core, shell and cloud genes are non-randomly distributed on Chr 1: An hypothesis that links the genomic location of genes with their intracellular placement
}

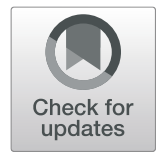

Cecilie Bækkedal Sonnenberg ${ }^{1}$, Tim Kahlke ${ }^{2}$ and Peik Haugen ${ }^{1 *}$ (D)

\begin{abstract}
Background: The genome of Vibrionaceae bacteria, which consists of two circular chromosomes, is replicated in a highly ordered fashion. In fast-growing bacteria, multifork replication results in higher gene copy numbers and increased expression of genes located close to the origin of replication of Chr 1 (ori1). This is believed to be a growth optimization strategy to satisfy the high demand of essential growth factors during fast growth. The relationship between ori7-proximate growth-related genes and gene expression during fast growth has been investigated by many researchers. However, it remains unclear which other gene categories that are present close to ori1 and if expression of all ori1-proximate genes is increased during fast growth, or if expression is selectively elevated for certain gene categories.
\end{abstract}

Results: We calculated the pangenome of all complete genomes from the Vibrionaceae family and mapped the four pangene categories, core, softcore, shell and cloud, to their chromosomal positions. This revealed that core and softcore genes were found heavily biased towards orit, while shell genes were overrepresented at the opposite part of Chr 1 (i.e., close to ter1). RNA-seq of Aliivibrio salmonicida and Vibrio natriegens showed global gene expression patterns that consistently correlated with chromosomal distance to ori1. Despite a biased gene distribution pattern, all pangene categories contributed to a skewed expression pattern at fast-growing conditions, whereas at slow-growing conditions, softcore, shell and cloud genes were responsible for elevated expression.

Conclusion: The pangene categories were non-randomly organized on Chr 1, with an overrepresentation of core and softcore genes around ori1, and overrepresentation of shell and cloud genes around ter1. Furthermore, we mapped our gene distribution data on to the intracellular positioning of chromatin described for $V$. cholerae, and found that core/softcore and shell/cloud genes appear enriched at two spatially separated intracellular regions. Based on these observations, we hypothesize that there is a link between the genomic location of genes and their cellular placement.

Keywords: Pangenome, Genome architecture, Vibrionaceae, Aliivibrio salmonicida, Vibrio natriegens, Gene dosage

\footnotetext{
*Correspondence: peik.haugen@uit.no

'Department of Chemistry and Center for Bioinformatics (SfB), Faculty of

Science and Technology, UiT The Arctic University of Norway, N-9037

Tromsø, Norway

Full list of author information is available at the end of the article
}

(c) The Author(s). 2020 Open Access This article is licensed under a Creative Commons Attribution 4.0 International License, which permits use, sharing, adaptation, distribution and reproduction in any medium or format, as long as you give appropriate credit to the original author(s) and the source, provide a link to the Creative Commons licence, and indicate if changes were made. The images or other third party material in this article are included in the article's Creative Commons licence, unless indicated otherwise in a credit line to the material. If material is not included in the article's Creative Commons licence and your intended use is not permitted by statutory regulation or exceeds the permitted use, you will need to obtain permission directly from the copyright holder. To view a copy of this licence, visit http://creativecommons.org/licenses/by/4.0/ The Creative Commons Public Domain Dedication waiver (http://creativecommons.org/publicdomain/zero/1.0/) applies to the data made available in this article, unless otherwise stated in a credit line to the data. 


\section{Background}

Bacteria that belong to the family Vibrionaceae are rich in most aqueous habitats, from the deep seas to fresh and brackish waters, and in temperature zones ranging from the polar to tropical areas. They exist as freeswimming cells or associated with other organisms, either in a symbiotic relationship or as pathogens of e.g. fish, corals and even humans $[1,2]$. Despite the notorious reputation of some Vibrionaceae species, (e.g., Vibrio cholerae and Vibrio vulnificus) it is the diversity of non-pathogenic Vibrionaceae species that makes these bacteria so successful and ecologically important [3]. The facultative anaerobic bacterium Vibrio natriegens, for example, fixes atmospheric nitrogen $\left(\mathrm{N}_{2}\right)$ into ammonia $\left(\mathrm{NH}_{3}\right)$, and thus provides its surroundings with a critical nutrient [4].

As of April 2020, the RefSeq database contains 306 complete Vibrionaceae genomes (representing 57 species), with genomes from new species being added on a regular basis. One characteristic feature shared by almost all Vibrionaceae genomes is a highly unusual bipartite structure consisting of a large (Chr 1) and a smaller (Chr 2) chromosome [5, 6]. It is proposed that bacteria with bipartite genomes have a selective advantage for the adaptation to very different environmental conditions [7], and that division into multiple smaller replicons may reduce replication time, thus allowing for faster generation time and a competitive advantage [8, 9]. The unconventional genome constellation is expected to require tightly regulated and synchronized replication to ensure proliferation and control of gene expression during changes in the surrounding environment.

In $V$. cholerae, replication of Chr 1 and Chr 2 is highly coordinated [10]. When the replication fork approaches crtS in Chr 1 (Chr 2 replication triggering site), a hitherto unknown mechanism triggers replication of Chr 2 $[11,12]$. Interestingly, there is a short pause (corresponding to replication of approx. $200 \mathrm{kbp}$ ) between the crtS replication and the initiation of Chr 2 replication. The exact function of this pause is yet unknown, but it is hypothesized to be needed for activation of the $\operatorname{rct} B$ (Chr 2's own replication initiator) and ori2 initiation system [12]. In other words, the chromosomal position of crtS and the pause contribute to synchronize termination of Chr 1 and Chr 2 replication. Furthermore, the synchronized termination is likely linked to coordination of chromosome segregation and cell division [12].

Another intriguing phenomenon regarding replication of Vibrio genomes is that genes surrounding ori can be found in multiple copies during the replication process due to successive initiations of replication from ori (i.e., multifork replication) $[13,14]$. This phenomenon is a hallmark of fast-growing bacteria, such as $V$. cholerae and $V$. natriegens, and is believed to be a growth optimization strategy to satisfy the high demand of essential growth factors during fast growth [15-17]. Using an elegant genetic approach, Soler-Bistué et al. (2015) showed that by relocating the major ribosomal protein gene locus $($ s10-spec- $\alpha)$ of $V$. cholerae further away from ori1, growth rate, the gene copy number and mRNA abundance of this cluster were reduced [18]. The authors concluded that there is a strong correlation between chromosomal gene position and effects on the bacterial physiology. Later, the same model system (i.e., $V$. cholerae with relocated $s 10$-spec- $\alpha$ locus) was used to study effects on bacterial fitness under slow growth conditions (i.e., no multifork replication) [19]. One conclusion from this study was that bacterial fitness was reduced when the 10 -spec- $\alpha$ locus was located distal to ori1, which demonstrates that genomic positioning of ribosomal protein genes not only affects growth, but also cell fitness across the whole life cycle. In a recent study, Soler-Bistue et al. (2020) showed that relocation of the s10-spec- $\alpha$ locus lead to higher cytoplasm fluidity and the authors suggested that changes in the macromolecular crowding of the cytoplasm impacts the cellular physiology of $V$. cholerae. Interestingly, the protein production capacity in $V$. cholerae was independent of the position of the $s 10$-spec- $\alpha$ locus [20].

In an interesting approach, Dryselius et al. (2008) used qPCR and microarray to study how copy numbers of genes vary across the entire genome of several Vibrio species ( $V$. parahaemolyticus, $V$. cholerae and $V$. vulnificus) under different growth conditions, and then monitored how the data correlated with gene expression levels (also using microarray) [21]. The authors found greatest differences in gene copy numbers across Chr 1 compared to Chr 2 when grown in a rich medium. In general, the trend is that gene copy numbers increase from the terminus towards the origin of replication, and that this increase is reflected by increasing gene expression levels. The same trend was not found for slowgrowing bacteria (i.e., when grown in minimal medium). Also, for Chr 2 gene expression levels were low and apparently independent of gene copy number effect. Similar findings were later described in V. splendidus [22]. Here, genes located on Chr 1 were $3.6 \times$ more expressed compared to those located on Chr 2, and the highest expression values were typically associated with genes surrounding the origin of replication on Chr 1.

In summary, the genome of Vibrionaceae bacteria, which consists of two circular chromosomes, is replicated in a highly ordered fashion. In fast-growing bacteria, replication results in higher gene copy numbers, and increased expression of genes located close to the origin of replication of Chr 1 . That the expression of growth-related genes located close to ori1 is elevated during fast growth is known, but a general picture of 
which gene types are found close to ori1, and how expression of each gene type is affected, is however not known. To address this knowledge gap we revisited the intriguing topic of genome architecture in Vibrionaceae. In a pangenome approach we used available genomes to calculate and divide clusters of orthologous genes into the main categories "core", "softcore", "shell" (accessory) and "cloud" (unique), and used this information to determine how the corresponding genes are distributed on Chr 1 and Chr 2 of selected Vibrionaceae genomes. Data from publicly available gene expression experiments was mapped back to the pangenes to determine gene expression profiles under different environmental conditions such as expression data from the fast-growing bacterium $V$. natriegens grown under optimal or minimal growth conditions, and data from the fish-pathogen Aliivibrio salmonicida grown under salt concentration and temperature that mimics the physiological conditions during infection. Our results show a non-random distribution of genes on the two chromosomes of Vibrionaceae. The gene distribution was then compared with global gene expression trends, and we find a strong correlation between expression levels and distance from ori1. Surprisingly, despite a biased gene distribution pattern, all pangene categories contribute to a skewed expression pattern at fast-growing conditions. Finally, based on our data we propose an hypothesis that describes how pangenes are spatially distributed inside Vibrionaceae bacterial cells, and we discuss possible implications of the proposed hypothesis.

\section{Results}

\section{Pangenome calculations based on 124 complete Vibrionaceae genomes identifies 710 clusters of orthologous core genes}

To categorize all genes associated with Vibrionaceae genomes into distinct classes, we downloaded all complete genomes from the NCBI RefSeq database (124 as of May 2018, see Additional file 1), and then used GET_HOMOLOGUES v3.1.0 [23] to cluster orthologous protein sequences based on the OrthoMCL algorithm. The pangenome calculations identified a total of 61,512 clusters, of which 710 were encoded by genes found in all 124 genomes (i.e., core genes). The remaining clusters are distributed among softcore (encoded by $\geq 117$ genomes), shell (encoded by $116 \leq$ and $\geq 3$ genomes) and cloud (encoded by $\leq 2$ genomes), and contain 1796, 14,642 and 45,074 clusters, which represents 3,23 and $73 \%$ of the total clusters, respectively. In individual genomes, core gene clusters represent $1.2 \%$ of the pangenome, and comprise $10-17 \%$ of the total genes. Similarly, softcore constitutes $24-34 \%$ (1489-1796 genes per genome) of the total genes.

\section{Core and softcore genes densely populate the upper half} of Chr 1

The four gene categories core, softcore, shell and cloud, were next mapped to their chromosomal locations to investigate whether they are randomly or non-randomly distributed on each chromosome. First, genes of eleven selected Vibrionaceae representatives (see Additional file 2 for phylogeny of the 11 genomes) were classified as either upper or lower (i.e., upper or lower half of the chromosome) based on their chromosomal location on Chr 1 and Chr 2 in relation to their distance of the origin of replication. As presented in Fig. 1 (complete table of pangene distribution is available as Additional file 3 and chi-squared test is available as Additional file 4), core and softcore genes are significantly overrepresented (adjusted chi-square $P$-value $\leq 0.05$ ) in the upper half of Chr 1 in all investigated genomes. Similarly, shell and cloud genes on Chr 1 are significantly overrepresented (adjusted chi-square P-value $\leq 0.05$ ) in the lower half of Chr 1 in 8 genomes, thus supporting a non-random distribution of genes on Chr 1 . In contrast to Chr 1 , genes of all categories are much more evenly distributed on Chr 2. Although shell, cloud and softcore genes show non-random distribution on Chr 2 in some of the investigated genomes (softcore $3 / 11$, shell $1 / 11$, cloud $2 / 11$ ), the majority of genomes show no significant bias (adjusted chi-square $P$-value $\leq 0.05$ ). Furthermore, core genes were not significantly overrepresented in either lower or upper half of Chr 2 in any of the genomes.

To provide a more fine-grained picture of the core (710-721) and shell (749-2753) gene distributions, we plotted the distribution of core and shell genes on Chr 1 and Chr 2 of eleven Vibrionaceae taxa using the genome comparison tool Circos [24] (Fig. 2). Each plot was centered on mioC (Chr 1) and $r c t B(C h r ~ 2)$. Our results show that although the exact distribution pattern varies between species, the biased distributions of core and shell, as described above, are striking and readily visible with the naked eye. Interestingly, although core genes densely populate the upper half of Chr 1 , the region immediately surrounding ori1 contains very few core genes. This region (denoted "i" in Fig. 2) is, in contrast, densely populated by softcore genes (at least in $V$. natriegens and A. salmonicida, see section below). Also, a region (denoted "ii" in Fig. 2) of approximately $500 \mathrm{~kb}$ surrounding ter 1 is densely populated with shell genes (and hence sparsely populated with core genes). For Chr 2, the chisquare test supported no significant bias in gene distribution (Additional file 4), and Fig. 2b supports this general picture although some local clustering of gene categories will occur. In summary, the results presented here reveal that core, softcore, shell and cloud genes are non-randomly distributed on Chr 1 . Core and softcore 

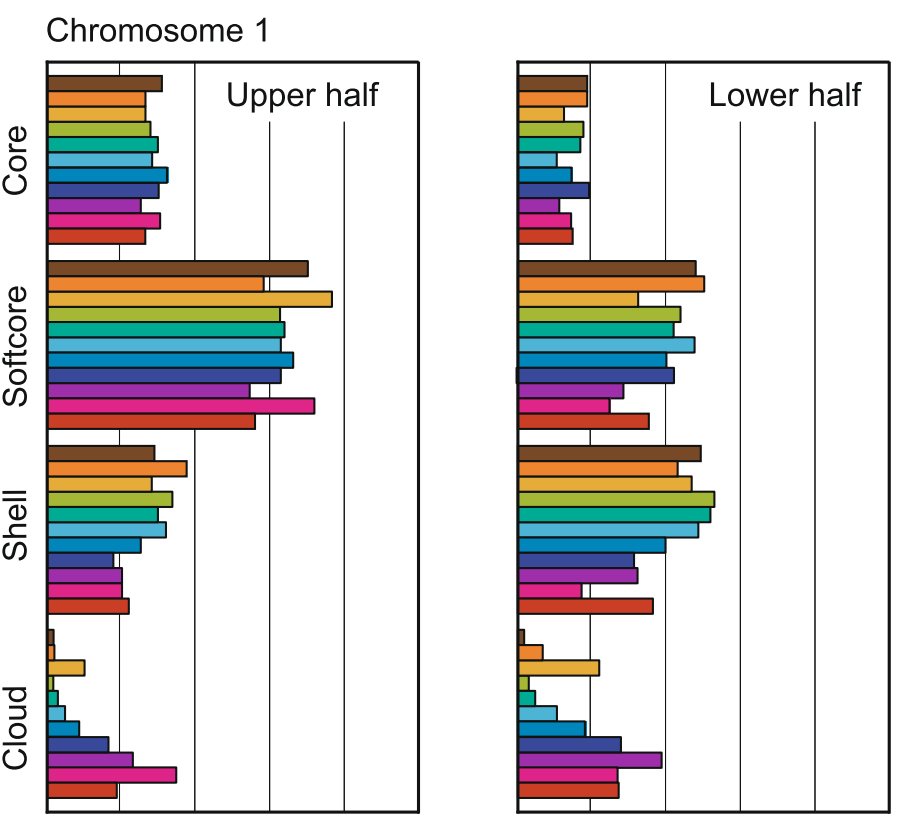

Chromosome 2
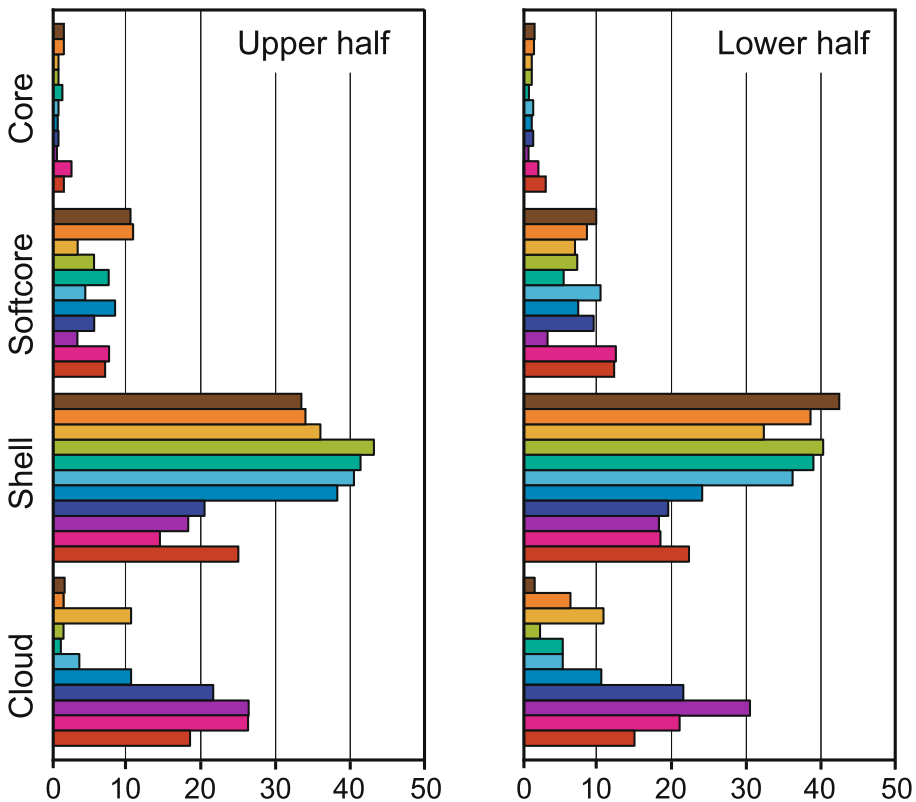

Percent of total CDS per chromosome

V. cholerae biovar El Tor str. N16961

V. anguillarum NB10

V. campbelli ATCC BAA-1116

V. parahaemolyticus RIMD 2210633

V. natriegens ATCC 14048

$\square$ V. vulnificus ATCC 27562
V. tasmaniensis LGP32

P. damselae KC-Na-1

P. profundum SS9

G. hollisae ATCC 33564

A. salmonicida LFI1238

Fig. 1 (See legend on next page.) 
(See figure on previous page.)

Fig. 1 Distribution of the four pangene categories between upper and lower half of 11 Vibrionaceae genomes. Bars in the histogram show percent of total CDSs per chromosome for each pangene category. Core and softcore genes are overrepresented on the upper half of Chr 1 , shell and cloud genes are overrepresented on the lower half. On Chr 2 the genes are more evenly distributed between the upper and lower halves of $\mathrm{Chr} 2$

genes are more likely to be located on the upper half of Chr 1, whereas shell and cloud genes tend to be located closer to the replication terminator. For Chr 2, the distribution of the four pangene categories are in general randomly distributed showing locational bias only for a few genomes.

\section{Expression levels of genes located on Chr 1 of $V$. natriegens and $A$. salmonicida generally correlate with distance to ori1}

Figure 3 shows how core, softcore, shell and cloud pangenes are distributed on Chr 1 and Chr 2 of $V$. natriegens and A. salmonicida. The pattern is consistent with

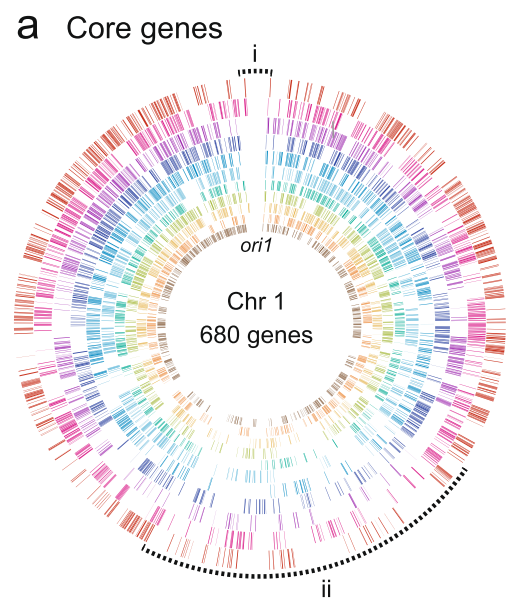

\section{b Shell genes}
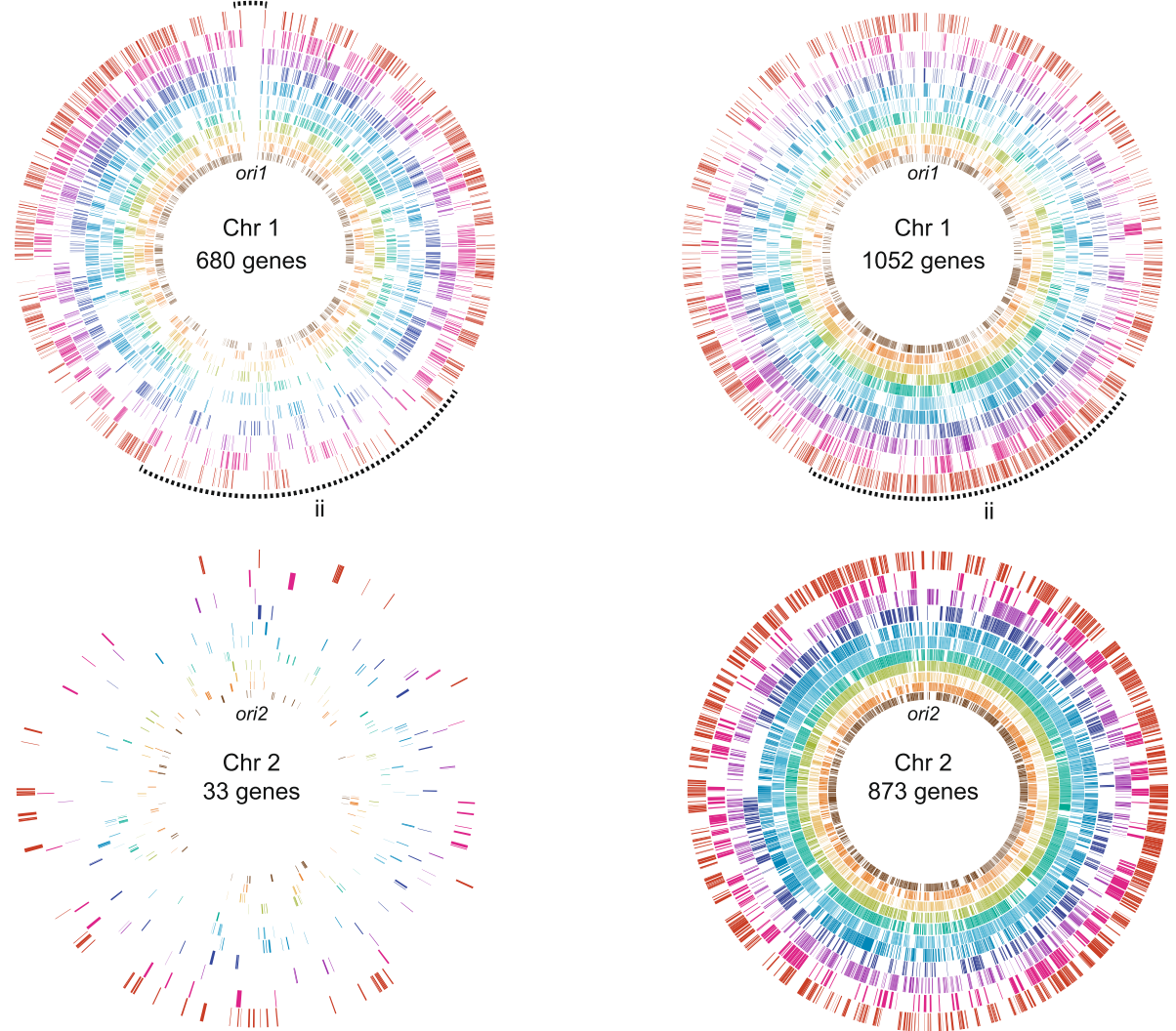

V. natriegens ATCC 14048

P. profundum SS9

V. vulnificus ATCC 27562

V. tasmaniensis LGP32

P. damselae KC-Na-1

Fig. 2 Distribution of 710 core genes in 11 Vibrio genomes. Location of core (a) and shell (a) genes on Chr 1 and Chr 2 of 11 Vibrionaceae genomes. Circular plots are arranged regarding the phylogenetic relationship of the investigated isolates. Each plot is centered at a gene assumed to be close to the replication origin: mioC on Chr 1 and rtcB on Chr 2. As shown, a majority of core genes on Chr 1 is located closer to ori than to ter. Shell genes show the opposite distribution pattern on Chr 1, where majority of shell genes accumulate closer to ter. On Chr 2 both core and shell genes are randomly distributed. The dashed line "i" indicates a region on Chr 1 surrounding ori1 that contains very few core genes. The dashed line "ii" shows a region on Chr 1 of approximately $500 \mathrm{~kb}$ surrounding ter that is more sparsely populated with core genes than the rest of the chromosome 

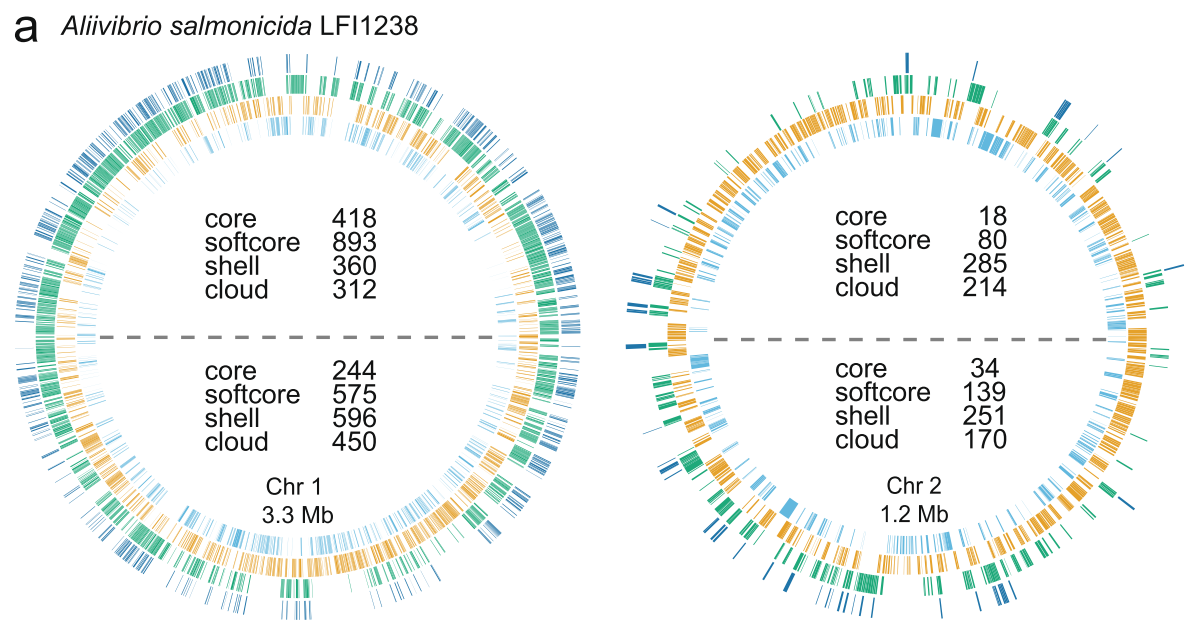

b Vibrio natriegens ATCC 14048
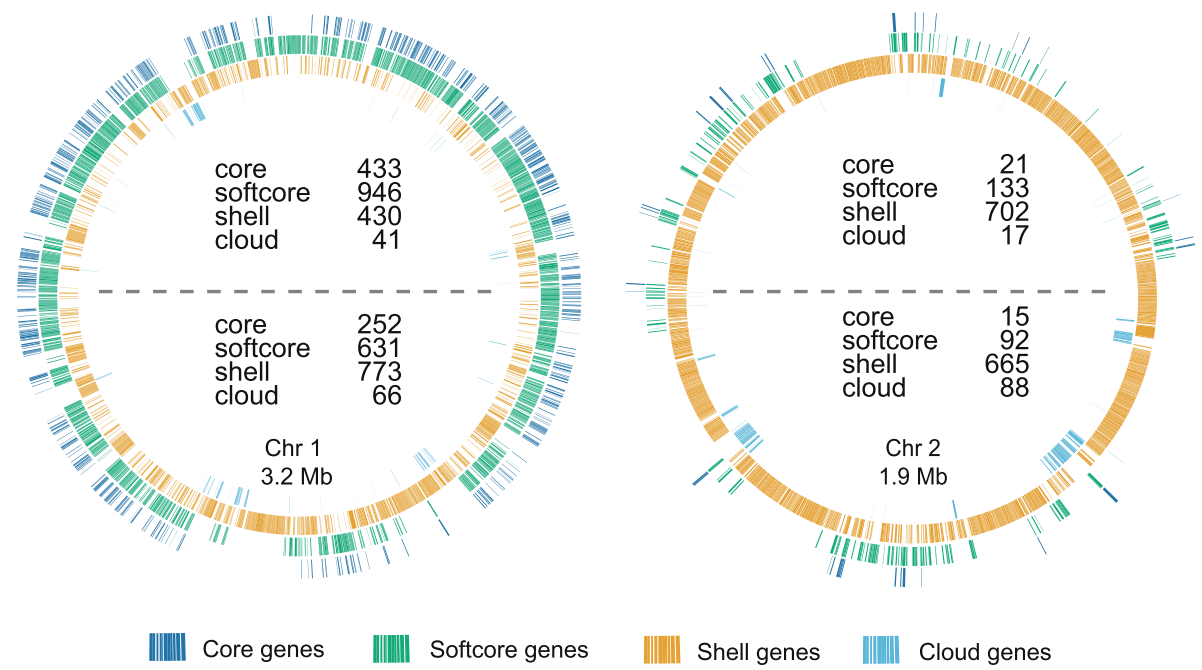

Fig. 3 Distribution of the four pangene categories on Chr 1 and Chr 2 for (a) A. salmonicida LFI1238 and (b) V. natriegens ATCC 14048. The number of genes in each pangene category in the upper and lower half is written inside each chromosome. A dashed line visualises the separation of the upper and lower half of the chromosomes

the biased gene distribution pattern described above, with core and softcore genes being overrepresented at the upper half of Chr 1, and shell and cloud genes being overrepresented at the lower half. The two species were chosen as models for comparison of gene expression data with pangene distribution patterns. Specifically, we were curious to examine if regions that are densely populated by core/softcore pangenes are expressed at high levels, compared to regions more sparsely populated by core/softcore pangenes. This expectation is based on previous data from $V$. parahaemolyticus and $V$. cholerae, which showed that growth rates have large impacts on the copy number (gene dosage) of genes located on $\mathrm{Chr}$ 1 , as well as on gene expression levels $[9,10,21]$. Fastand slow-growing bacterial representatives were therefore chosen for this particular comparative analysis. $V$. natriegens is a fast-growing bacterium commonly found in estuarine mud, with doubling times below $10 \mathrm{~min}$ at favourable conditions [25]. A. salmonicida is, in contrast, a slow growing Vibrionaceae bacterium, and the causative agent of cold-water vibriosis in e.g., Atlantic salmon and cod [26, 27]. To correlate gene distribution with gene expression data, publicly available RNA-seq data of $V$. natriegens and $A$. salmonicida were downloaded from the Sequence Read Archive [28] at NCBI. For V. natriegens, datasets from growth in minimal and optimal (rich) medium at $37^{\circ} \mathrm{C}$ to mid log phase were chosen [29]. For A. salmonicida, a dataset originating from growth in LB medium containing $1 \% \mathrm{NaCl}$ at $8^{\circ} \mathrm{C}$ to mid log phase was used [30]. EDGE-PRO 1.3.1 [31] was used to align cDNA reads to the $V$. natriegens ATCC 14048 (NBRC 15636, DSM 759) (assembly no. GCA_001456255.1) or 
A. salmonicida LFI1238 (assembly no. GCF_ 000196495.1) genome, and to calculate expression values as reads per kilobase per million (RPKM) for all protein coding sequences (CDS).

Figure 4 shows global expression maps of $V$. natriegens and $A$. salmonicida chromosomal genes centered around the median. Data points $\left(\log _{2}\right.$ ratio RPKM CDS:RPKM median) for each CDS are shown, as well as a trend line averaged over a sliding window of 200 data points. For Chr 1 the general picture is similar in all three datasets, i.e., RPKM values are typically above the median value at the upper half (i.e., the region closest to the origin of replication), but lower at the region surrounding the terminus, independent of growth conditions. This is somewhat surprising since the observed expression patterns described above was expected for fast growing cultures (i.,e $V$. natriegens in rich medium), but not for slow growing cultures (i.e., A. salmonicida in LB $1 \%$ $\mathrm{NaCl}$ and $8{ }^{\circ} \mathrm{C}$ and $V$. natriegens in minimal medium, see Additional file 5). The rationale is that gene copy numbers (also known as "gene dosage"), and thus expression levels are expected to be correlated with growth rates/multifork replication [21].

A more detailed circular expression map is available in Additional file 6 and shows that region "i" (see Fig. 2), which encodes mostly softcore genes, contains a highly expressed proton-translocating ATP synthase $\left(\mathrm{F}_{0} \mathrm{~F}_{1}\right.$ class) gene cluster (atpIBEFHAGDC). The ATPase cluster is well described in Escherichia coli as an operon located $84 \mathrm{~min}$ on the chromosome (close to oriC), and with gene expression levels varying according to cell growth rate [32]. The ATP synthase cluster represents softcore genes, and are present in both bacteria. Moreover, the detailed map shows that region "ii", which is densely populated with shell genes, differs from the remaining lower half of Chr 1 by being expressed far below median in $V$. natriegens at both fast and slow growth conditions. For $A$. salmonicida the main picture is the same, but less pronounced, meaning that the majority of shell genes located in "ii" are expressed below median.

For Chr 2, the results are more ambiguous, although overall similar between minimal and rich growth. For A. salmonicida, expression around the terminus is, on average, higher compared to that of regions adjacent to ori2. For $V$. natriegens, expression is generally higher than median in regions surrounding the terminus, but varies across the remaining parts of Chr 2. Similar to Chr 1, little difference could be determined between the slow- and the fastgrowing datasets of Chr 2 .

In summary, we found that global expression levels for Chr 1, consistently correlate with the distance to the origin of replication. The $\log 2$ ratio of RPKM CDS:RPKM median decreases as the distance from origin of replication increases. a Aliivibrio salmonicida LFI1238

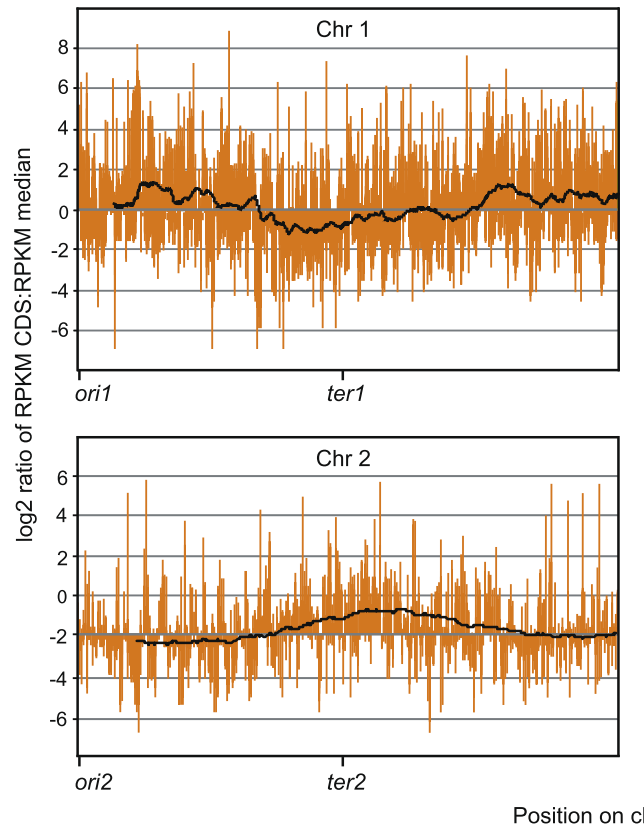

b Vibrio natriegens ATCC 14048
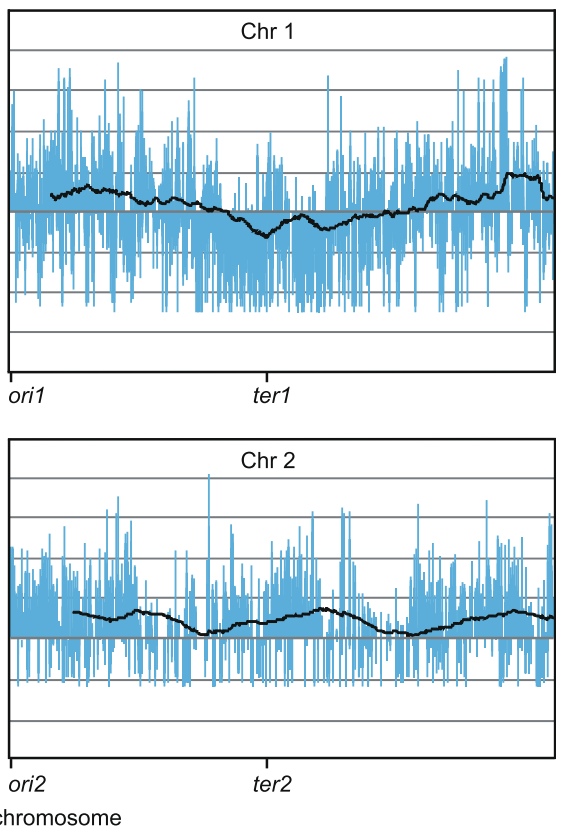

Fig. 4 Global expression maps of (a) A. salmonicida LFI1238 and (b) V. natriegens ATCC 14048 chromosomal genes centered around the median. Data points ( $\log _{2}$ ratio RPKM CDS:RPKM median) for each CDS are shown, as well as a trend line averaged over a sliding window of 200 data points. V. natriegens ATCC 14048 is grown under fast-growing conditions and A. salmonicida LFI1238 is grown under suboptimal conditions 


\section{All pangene categories contribute to higher expression levels around ori1 at fast-growth conditions, but not at slow-growth conditions}

The global trend described above can be explained by generally higher expression levels of all pangene categories located close to ori1, or, higher expression of three or less of the four pangene categories. To discriminate between the two alternatives, we calculated the RPKM median value for each pangene category, and compared the median values for genes located on the upper or lower halves of Chr 1 (Table 1). The Wilcoxon signed-rank test strongly support $(\mathrm{P}$-adj $\leq 0.05)$ that median values for all four pangene categories are significantly higher for genes located on the the upper half, i.e., when $V$. natriegens is cultured at fast-growth ("optimal") conditions. Notably, when grown under slow-growing conditions, median values for softcore, shell and cloud genes located on the upper half are significantly higher. Core genes are in contrast, expressed at equal levels on both halves. This applies for both $V$. natriegens (RPKM median $=370$ and $360, P$-adj $=0.321)$ in minimal medium, and $A$. salmonicida (RPKM median $=301$ and 309, $P$ $\operatorname{adj}=0.717)$ at suboptimal conditions. Conversely, we can therefore state that genes from all pangene categories located on the lower half are generally expressed at lower levels compared to those on the upper half (except for core genes at slow growth conditions). To summarize, we conclude that gene expression levels correlate with distance to oril (Fig. 4), and genes from all four pangene categories contribute to this trend when grown under fast-growing conditions, whereas softcore, shell and cloud genes contribute at slow-growing conditions.

\section{Discussion}

Inspired by the discovery of multifork replication and increased copy numbers of genes surrounding the origin of replication, researchers have for decades studied how different categories of genes are distributed on chromosomes and at which level these genes are expressed. Here, we revisited this topic and describe hitherto hidden/unrecognized global gene distribution and expression patterns in Vibrionaceae. First, we mapped pangenes to their chromosomal positions and revealed that core and softcore genes are found heavily biased towards the ori1 of Chr 1 . Shell genes are, in contrast, overrepresented at the opposite part of Chr 1 (i.e., close to ter). We next found that gene expression strongly correlates with chromosomal distance to ori1. This trend is caused by higher expression of all pangene categories at fast-growing conditions, whereas softcore, shell and cloud genes are responsible for biased (higher) expressing on the upper half of Chr 1 at slow-growing conditions.

\section{Pangene categories are non-randomly distributed on Chr 1} In this work we report a clear pattern where core/softcore genes are overrepresented on the upper half of Chr 1 of Vibrionaceae, particularly at regions corresponding to 10 11 and 1-2 O'clock on Chr 1, and shell/cloud genes are overrepresented in the ter 1 region (Fig. 2). In comparison, no clear pattern was recorded for Chr 2, i.e., the distribution of pangenes appear generally independent of location. For Chr 1, the core/softcore gene distribution pattern resembles that described for genes involved in translation and transcription in E. coli $[16,17,33]$ and in several Vibrio species $[16,17,21]$. More precisely, Couturier and

Table 1 Comparison of gene expression levels for pangenes located on the upper or lower halves of Chr 1

\begin{tabular}{|c|c|c|c|c|c|c|c|c|c|c|c|c|}
\hline & \multicolumn{4}{|c|}{ A. salmonicida } & \multicolumn{4}{|c|}{ V. natriegens slow-growth } & \multicolumn{4}{|c|}{ V. natriegens fast-growth } \\
\hline & core & softcore & shell & cloud & core & softcore & shell & cloud & core & softcore & shell & cloud \\
\hline \multicolumn{13}{|l|}{ Upper halfa } \\
\hline $\mathrm{Q}_{1}$ & 152 & 118 & 42 & 42 & 188 & 126 & 21 & 5 & 249 & 170 & 36 & 37 \\
\hline $\mathrm{Q}_{2}$ & 301 & 245 & 89 & 67 & 370 & 288 & 71 & 147 & 447 & 341 & 93 & 269 \\
\hline $\mathrm{Q}_{3}$ & 853 & 633 & 197 & 197 & 1101 & 760 & 190 & 426 & 1059 & 719 & 241 & 581 \\
\hline Max & 34,254 & 34,254 & 6473 & 13,656 & 23,238 & 23,238 & 17,161 & 5533 & 35,274 & 35,274 & 28,737 & 4049 \\
\hline \multicolumn{13}{|l|}{ Lower half ${ }^{a}$} \\
\hline $\mathrm{Q}_{1}$ & 151 & 89 & 34 & 25 & 143 & 83 & 4 & 4 & 178 & 109 & 0 & 0 \\
\hline $\mathrm{Q}_{2}$ & 309 & 207 & 65 & 47 & 360 & 192 & 28 & 18 & 328 & 232 & 26 & 17 \\
\hline $\mathrm{Q}_{3}$ & 695 & 486 & 133 & 82 & 966 & 565 & 74 & 59 & 696 & 480 & 97 & 62 \\
\hline Max & 53,501 & 8098 & 19,837 & 23,646 & 14,116 & 14,116 & 15,800 & 463 & 16,521 & 17,549 & 17,550 & 535 \\
\hline$P$-value $Q_{2}{ }^{b}$ & 0.71 & 0.01 & 0.00 & 0.00 & 0.32 & 0.00 & 0.00 & 0.00 & 0.00 & 0.00 & 0.00 & 0.00 \\
\hline
\end{tabular}

${ }^{a} Q_{1}$ is the RPKM value at the first quartile. $Q_{1}$ is defined as the middle number between the smallest number and the median (i.e., the second quartile $Q_{2}$ ), if the data numbers (in this case RPKM values) are ordered from smallest to largest. The third quartile $\left(Q_{3}\right)$ is the middle value between the median $\left(Q_{2}\right)$ and the maximum (Max) value

${ }^{\mathrm{b}}$ Adjusted $P$-values from Wilcoxon signed-rank test, to test if $\mathrm{Q}_{2}$ values (median) of genes located on the upper half of Chr 1 are significantly different from $\mathrm{Q}_{2}$ values of genes located on the lower half. Values below 0.05 are considered significant 
Rocha (2006) showed that genes involved in translation and transcription in four Vibrio species are typically found close to ori1 of Chr 1. Chr 2 contained, in contrast, fewer genes related to translation and transcription than would be expected. Iida and coworkers [21] later found that genes related to growth (both essential and contributing) are located in close proximity to ori1 in $V$. cholerae. Overrepresentation of core/softcore genes, many of which are important for growth, at the region proximate to ori1 of Vibrionaceae Chr 1 can be explained by an increase in demand for ori1-proximate gene products during fast growth (i.e., multifork replication results in elevated gene copy numbers and increased transcription levels). For example, genes that encode ribosomal RNA and ribosomal proteins are found clustered in the upper half of Chr 1 , and are expressed at extremely high levels, which support this hypothesis.

Moreover, we found that during fast growth of $V$. natriegens, core, softcore, shell and cloud genes are all expressed at higher levels on the upper half of the chromosome compared to the lower half. In slowgrowing $V$. natriegens and $A$. salmonicida, only softcore, shell and cloud genes followed the same trend, which suggests that regulatory mechanisms other than "gene dosage" are in play, to ensure a relatively low and uniform expression of core genes independent of chromosomal position during slow growth.

\section{Why are core and softcore genes clustered at the old pole area of cells?}

It is well documented in the literature that the intracellular space of bacteria is highly organized, with defined structures at specific locations (reviewed by Surovtsev and Jacobs-wagner 2019) [34]. For example, Chr 1 and Chr 2 of $V$. cholerae are spatially organised in a longitudinal orientation inside the cell, with their chromatin stretching from one pole to the other. ori1 and ter 1 of Chr 1 are located at the old and new poles, respectively, whereas ori 2 and ter 2 of Chr 2 stretches from the new pole towards the cell's center, respectively (Fig. 5). The organization of Chr 1 and Chr 2 in $V$. cholerae has been established by both fluorescence tag microscopy $[9,35,36]$ and chromosome conformation capture (3C) [11]. In the light of this knowledge, our data then suggest that core/ softcore and shell/cloud genes are enriched at two spatially separated intracellular regions, i.e., at the two extreme poles of Vibrionaceae cells, given that the spatial positioning of chromatin described for $V$. cholerae applies to all representatives within the family. We emphasize, however, that this hypothesis is based on limited data and should be further tested in future experiments before any strong conclusion can be made. Below we further speculate on why core and softcore genes appear clustered at the old (flagellated) pole area.

Given a non-random structural organization of the genes (as hypothesized above), this then suggests to us that there is a link between gene placement and their function, and that the underlying reasons for the strong distribution pattern could be very complex. The full complexity of factors that affects gene expression can be illustrated by e.g., chromatin packing [37-41], nucleoidassociated proteins (NAPs) [42-44], Structural Maintenance of Chromosome complex (SMC) [45], RNA polymerase (RNAP) [46-50], transcription factors and promoter strength/chromosomal position [43, 51] and macromolecular crowding [20]. Perhaps the most fundamental factor is chromatin packing and organization. The density of chromatin is determined by a number of circumstances, including differential abundance/availability of macromolecular machineries [38, 41, 46-50, 52, 53]. In this respect the bipartite DNA organization of Vibrionaceae represents a special case because Chr 1 stretches from pole to pole, whereas Chr 2 prolongates from the new pole towards the cell center, thus suggesting that the chromatin density varies between the two halves of the cell. Higher chromatin density will presumably reduce the diffusion of
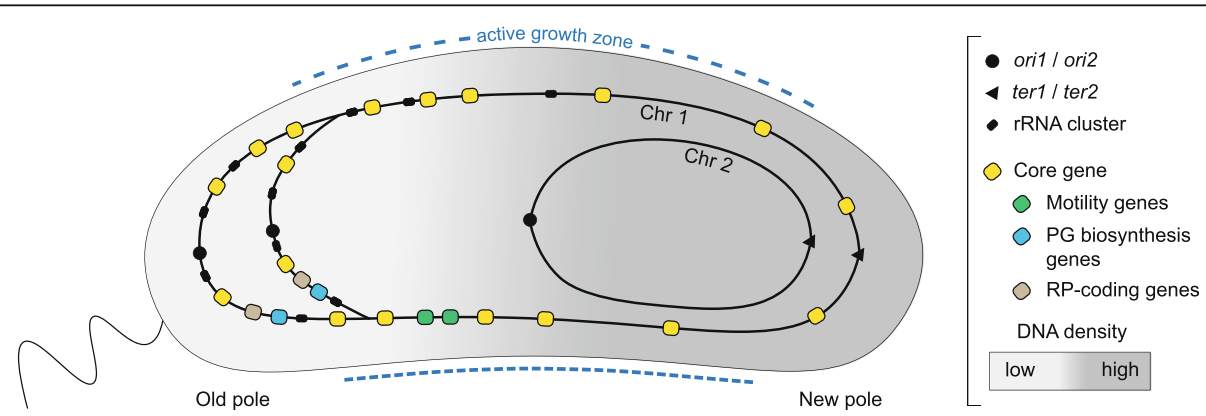

Fig. 5 Subcellular distribution of Chr 1 and Chr 2 in V. cholerae. Core genes are spatially enriched in the intracellular region near the old pole. Coloured core gene clusters (related to motility, peptidoglycan biosynthesis and ribosomal proteins) represent core gene products that colocalize with growth/survival-related reactions in the old pole of the cell. Two replication origins on Chr 1 indicate multifork replication. Active growth zones are indicated with blue dashed lines along the axis of the cell. Small dashes illustrate fast peptidoglycan growth and long dashes illustrate slower growth 
macromolecular particles, such as proteins and ribosomes, in the nucleoid/DNA meshwork. Given that the DNA density is lower in the old pole area, the extra cytoplasmic space will presumably result in increased diffusion and transport of gene products, which provides a plausible explanation for the high abundance of core genes (many of which are growth related), and also the ribosomal protein clusters and rRNA clusters, in this subcellular region. Production of core gene products will therefore coincide and co-localize with the greatest number of growth/survivalrelated reactions and processes in the cell. A number of such cases can be mentioned, albeit we highlight two potential cases below.

The insertion of peptidoglycan (PG) in the cell wall happens in a dispersed manner, with the active growth zones along the axis [54]. To form the inner curvature of Vibrio cells, PG insertion is biased along the outer curve. Genes involved in cell wall synthesis are located in close proximity to ori1 on $V$. cholerae Chr 1 , with the main gene cluster related to nascent PG synthesis positioned approximately $0.38 \mathrm{Mb}$ from ori1. This suggests that the first step of PG synthesis preferentially takes place in the old pole area. Similarly, motility related genes are found clustered $0.6 \mathrm{Mb}$ from ori1, which is spatially close to the flagellum at the old pole.

\section{Conclusions}

Our results show a non-random organization of pangene categories on the two chromosomes of Vibrionaceae, with an overrepresentation of core and softcore genes around ori1. Gene distribution was compared with global gene expression trends and showed that during fast growth, all pangene categories contribute to a skewed expression pattern in respect to ori1. From our data and previous literature, we can deduce that core and softcore genes are overrepresented at the old pole area of $V$. cholerae. We hypothesize that this pattern can be beneficial due to spatial links between the structural organization of core genes and their cellular function, and that differences in intracellular DNA densities might further contribute to the biased gene distribution. These findings add to the growing list of examples of spatial order in bacteria, and scientists will surely continue to study the interplay between genome organization, gene activity and cellular function. We envision to explore how different pangene categories are distributed on chromosomes of other bacterial orders, and to search for similar spatial links to gene functions to investigate if our current findings are part of a general trend in Bacteria, or specific to Vibrionaceae.

\section{Methods}

Genome retrieval and gene annotation

As of May 2018 a total of 124 complete Vibrionaceae genomes were publicly available at the National Center for
Biotechnology Information (NCBI) which were downloaded from the RefSeq database at NCBI [55] (see Additional file 1 for a complete list). All genome sequences were re-annotated using RAST (Rapid Annotation using Subsystem Technology) version 2.0 [56] with default settings. The annotation of the 124 genome sequences resulted in a total of 555,513 annotated protein sequences.

\section{Pangenome approach to extract core, softcore, shell and cloud genes from large genome dataset}

To categorize the annotated Vibrionaceae protein sequences into four categories (core, softcore, shell and cloud genes) we performed pangenome analysis using the software package GET_HOMOLOGUES (v3.1.0 (20180103)) [23]. The clustering algorithm OrthoMCL was used to cluster homolog protein sequences. The parameter "minimum percent sequence identity" was set to 50 and "minimum percent coverage in BLAST query/ subj pairs" was set to 75 (default).

\section{Comparison of core, softcore, shell and cloud genes from 11 species}

We chose 11 representative species (based on phylogeny and scientific interest i. e. number of papers published in PubMed) to study the distribution of core, softcore, shell and cloud genes on Chr 1 and Chr 2. Chr 1 and Chr 2 were divided into "upper half" (close to ori) and "lower half" (close to ter) and the number of core, softcore, shell and cloud genes in each half were counted (see Additional file 3). The 11 species were used to study the exact chromosomal positions of core and shell genes on Chr 1 and Chr 2. The DoriC database [57] was used to locate ori1 and ori2 in Chr 1 and Chr 2 to subsequently center the plotted chromosomes at origin of replication, respectively at mioC on $C h r 1$ and $r t c B$ on $C h r 2$. The software package Circos [24] was used to visualize the gene distributions on the chromosomes.

\section{Analysing gene expression: mapping of read files on reference genomes}

To study gene expression of core, softcore, shell and cloud genes in A. salmonicida LFI1238 and $V$. natriegens ATCC 14048 (NBRC 15636, DSM 759), the following datasets were downloaded from the Sequence Read Archive [28] at the NCBI: for $V$. natriegens ATCC 14048 datasets from growth in minimal (BioSample accession no. SAMN10926309, SAMN10926310 and SAMN10926313) and optimal (rich) medium (sample no. SAMN10926311, SAMN10926312 and SAMN10926329) at $37^{\circ} \mathrm{C}$ to $\mathrm{OD}_{600 \mathrm{~nm}}$ 0.3-0.5 [29]; for A. salmonicida LFI1238 one dataset (sample no. SAMEA4548122, SAMEA4548133, SAMEA4548134) originating from growth in LB medium containing $1 \% \mathrm{NaCl}$ at $8{ }^{\circ} \mathrm{C}$ to mid log phase $\left(\mathrm{OD}_{600 \mathrm{~nm}}\right.$ 
0.5) [30]. The salt concentration is expected to be similar to the concentration the bacterium would experience inside its natural host (Atlantic salmon), where the bacterium is known to cause cold water vibriosis at temperatures below $10^{\circ} \mathrm{C}[26,27]$. Hence, $8{ }^{\circ} \mathrm{C}$ was used in the experiment. The quality of the reads was checked using FastQC [58]. EDGE-pro v1.0.1 (Estimated Degree of Gene Expression in Prokaryotes) [31] in Galaxy was used to align cDNA reads to $V$. natriegens ATCC 14048 (assembly no. GCA_001456255.1) and A. salmonicida LFI1238 (assembly no. GCF_000196495.1) and estimate gene expression as reads per kilobase per million (RPKM) for all protein coding sequences (CDS). The RPKM values were then used to calculate the $\log _{2}$ ratio RPKM CDS: RPKM median to make global expression maps for each of the three datasets.

\section{Statistical analysis}

Statistical analysis was performed using $\mathrm{R}$ in RStudio. Significance of gene distribution on either the upper or lower half of the chromosomes was performed using R's chisq.test() function for the non-parameteric chi-squared test (see Additional file 4). Significance of gene expression between gene classes located on the upper or lower half of the chromosomes was performed using R's wilcox.test() function for unpaired Wilcoxon signed-rank tests (see Additional file 4 ). For both analyses $P$-values were Bonferroni corrected for multiple comparisons using R's p.adjust() function.

\section{Supplementary information}

Supplementary information accompanies this paper at https://doi.org/10. 1186/s12864-020-07117-5.

\section{Additional file 1: Table S1. Complete list of the 124 Vibrionaceae} genomes used in this study.

Additional file 2: Fig. S1. The 11 Vibrionaceae representatives mapped to a phylogeny.

Additional file 3: Table S2. Distribution and percent of total number of CDSs per chromosome of core, softcore, shell and cloud genes on «upper half» and «lower half» of Chr 1 and $\mathrm{Chr} 2$ of 11 representative Vibrionaceae genomes.

Additional file 4: Table S3. Statistical analysis of gene distribution (chisquared test) and gene expression (Wilcoxon signed-rank test) between "upper half" and "lower half" of Chr 1 and Chr 2.

Additional file 5: Fig. S2. Global expression maps of $V$. natriegens ATCC 14048 (grown under slow-growing conditions) chromosomal genes centered around the median. Data points ( $\log _{2}$ ratio RPKM CDS:RPKM median) for each CDS are shown, as well as a trend line averaged over a sliding window of 200 data points.

Additional file 6: Fig. S3. Circular visualization of pangene distribution and gene expression ( $\log _{2}$ ratio RPKM CDS:RPKM median) of (a) $A$. salmonicida LFI1238 and V. natriegens ATCC 14048 grown under (b) fastand (c) slow-growing conditions.

\section{Acknowledgements}

We thank Professor Tomoo Sawabe, Hokkaido University, for providing multiple sequence alignments.

\section{Authors' contributions}

$\mathrm{PH}$ and CBS designed the study and wrote the manuscript. CBS performed all bioinformatics analysis. TK did statistical analyses and contributed to the writing of the manuscript. All authors contributed to proofreading and approved on the final manuscript.

\section{Funding}

The publication charges for this article have been funded by a grant from the publication fund of UiT The Arctic University of Norway. The funder had no role in study design, data collection and analysis or preparation of the manuscript.

\section{Availability of data and materials}

All data analysed during this study are included in this published article, its additional files and publicly available repositories. The RNA-seq datasets used in this study are available at Sequence Read Archive at Bioproject Accession PRJNA522293 [29] and PRJEB17700 [30].

Ethics approval and consent to participate

Not applicable.

Consent for publication

Not applicable.

\section{Competing interests}

The authors declare that they have no competing interests.

\section{Author details}

'Department of Chemistry and Center for Bioinformatics (SfB), Faculty of Science and Technology, UiT The Arctic University of Norway, N-9037 Tromsø, Norway. ${ }^{2}$ Climate Change Cluster, University of Technology Sydney, Sydney, NSW, Australia.

Received: 9 June 2020 Accepted: 29 September 2020

Published online: 06 October 2020

\section{References}

1. Thompson FL, lida T, Swings J. Biodiversity of Vibrios. Microbiol Mol Biol Rev. 2004;68:403-31.

2. Takemura AF, Chien DM, Polz MF. Associations and dynamics of Vibrionaceae in the environment, from the genus to the population level. Front Microbiol. 2014;5:38

3. Montánchez I, Kaberdin VR. Vibrio harveyi: a brief survey of general characteristics and recent epidemiological traits associated with climate change. Mar Environ Res. 2020;154:104850.

4. Hoff J, Daniel B, Stukenberg D, Thuronyi BW, Waldminghaus T, Fritz G. Vibrio natriegens: an ultrafast-growing marine bacterium as emerging synthetic biology chassis. Environ Microbiol. 2020;10.

5. Okada K, lida T, Kita-Tsukamoto K, Honda T. Vibrios commonly possess two chromosomes. J Bacteriol. 2005;187:752-7.

6. di Cenzo GC, Finan TM. The Divided Bacterial Genome: Structure, Function, and Evolution. Microbiol Mol Biol Rev. 2017;81:e00019-7.

7. Val ME, Kennedy SP, El Karoui M, Bonné L, Chevalier F, Barre FX. FtsKdependent dimer resolution on multiple chromosomes in the pathogen Vibrio cholerae. PLoS Genet. 2008:4:e1000201.

8. Egan ES, Fogel MA, Waldor MK. MicroReview: divided genomes: negotiating the cell cycle in prokaryotes with multiple chromosomes. Mol Microbiol. 2005;56:1129-38.

9. Srivastava P, Chattoraj DK. Selective chromosome amplification in Vibrio cholerae. Mol Microbiol. 2007;66:1016-10289.

10. Rasmussen T, Jensen RB, Skovgaard O. The two chromosomes of Vibrio cholerae are initiated at different time points in the cell cycle. EMBO J. 2007; 26:3124-31.

11. Val ME, Marbouty M, de Lemos MF, Kennedy SP, Kemble H, Bland MJ, et al. A checkpoint control orchestrates the replication of the two chromosomes of Vibrio cholerae. Sci Adv. 2016;2:e1501914.

12. Kemter FS, Messerschmidt SJ, Schallopp N, Sobetzko P, Lang E, Bunk B, et al. Synchronous termination of replication of the two chromosomes is an evolutionary selected feature in Vibrionaceae. PLoS Genet. 2018;14:e1007251.

13. Cooper S, Helmstetter CE. Chromosome replication and the division cycle of Escherichia coli B/r. J Mol Biol. 1968;31:519-40. 
14. Stokke C, Waldminghaus T, Skarstad K. Replication patterns and organization of replication forks in Vibrio cholerae. Microbiology. 2011;157:695-708.

15. Slager J, Veening JW. Hard-wired control of bacterial processes by chromosomal gene location. Trends Microbiol. 2016;24:788-800.

16. Rocha EPC. The replication-related organization of bacterial genomes. Microbiology. 2004;150:1609-27.

17. Couturier E, Rocha EPC. Replication-associated gene dosage effects shape the genomes of fast-growing bacteria but only for transcription and translation genes. Mol Microbiol. 2006;59:1506-18.

18. Soler-Bistué A, Mondotte JA, Bland MJ, Val ME, Saleh MC, Mazel D. Genomic location of the major ribosomal protein gene locus determines Vibrio cholerae global growth and infectivity. PLoS Genet. 2015;11:e1005156.

19. Soler-Bistue A, Timmermans M, Mazel D. The proximity of ribosomal protein genes to oric enhances Vibrio cholerae fitness in the absence of multifork replication. MBio. 2017;8.

20. Soler-Bistué A, Aguilar-Pierlé S, Garcia-Garcerá M, Val M-E, Sismeiro O, Varet $\mathrm{H}$, et al. Macromolecular crowding links ribosomal protein gene dosage to growth rate in Vibrio cholerae. BMC Biol. 2020;18:43.

21. Dryselius R, Izutsu K, Honda T, lida T. Differential replication dynamics for large and small Vibrio chromosomes affect gene dosage, expression and location. BMC Genomics. 2008;9:559.

22. Toffano-Nioche C, Nguyen AN, Kuchly C, Ott A, Gautheret D, Bouloc P, et al. Transcriptomic profiling of the oyster pathogen Vibrio splendidus opens a window on the evolutionary dynamics of the small RNA repertoire in the Vibrio genus. RNA. 2012;18:2201-19.

23. Contreras-Moreira B, Vinuesa P. GET_HOMOLOGUES, a versatile software package for scalable and robust microbial pangenome analysis. Appl Environ Microbiol. 2013;79:7696-701.

24. Krzywinski M, Schein J, Birol I, Connors J, Gascoyne R, Horsman D, et al. Circos: an information aesthetic for comparative genomics. Genome Res. 2009;19:1639-45.

25. Eagon RG. Pseudomonas natriegens, a marine bacterium with a generation time of less than 10 minutes. J Bacteriol. 1962;83:736-7.

26. Colquhoun DJ, Sørum H. Temperature dependent siderophore production in Vibrio salmonicida. Microb Pathog. 2001;31:213-9.

27. Enger $\mathrm{O}$, Husevåg B, Goksøyr J. Seasonal variation in presence of Vibrio salmonicida and total bacterial counts in Norwegian fish-farm water. Can $J$ Microbiol. 1991;37:618-23.

28. Leinonen $R$, Sugawara $H$, Shumway $M$. The sequence read archive. Nucleic Acids Res. 2011:39:D19-21.

29. Lee HH, Ostrov N, Wong BG, Gold MA, Khalil AS, Church GM. Functional genomics of the rapidly replicating bacterium Vibrio natriegens by CRISPRi. Nat Microbiol. 2019;4:1105-13.

30. Thode SK, Bækkedal C, Söderberg JJ, Hjerde E, Hansen H, Haugen P. Construction of a fur null mutant and RNA-sequencing provide deeper global understanding of the Aliivibrio salmonicida Fur regulon. PeerJ. 2017;5: e3461.

31. Magoc T, Wood D, Salzberg SL. EDGE-pro: estimated degree of gene expression in prokaryotic genomes. Evol Bioinformatics Online. 2013;9:127-36.

32. Kasimoglu E, Park SJ, Malek J, Tseng CP, Gunsalus RP. Transcriptional regulation of the proton-translocating ATPase (atplBEFHAGDC) operon of Escherichia coli: control by cell growth rate. J Bacteriol. 1996;178:5563-7.

33. Ardell DH, Kirsebom LA. The genomic pattern of tDNA operon expression in E. coli. PLoS Comput Biol. 2005;1:0086-99.

34. Surovtsev I, Jacobs-Wagner C. Subcellular organization: a critical feature of bacterial cell replication. Cell. 2018;172:1271-93.

35. Fogel MA, Waldor MK. Distinct segregation dynamics of the two Vibrio cholerae chromosomes. Mol Microbiol. 2005;55:125-36.

36. David A, Demarre G, Muresan L, Paly E, Barre FX, Possoz C. The two cisacting sites, parS1 and oriC1, contribute to the longitudinal organisation of Vibrio cholerae chromosome I. PLoS Genet. 2014;10:e1004448.

37. Martis BS, Forquet R, Reverchon S, Nasser W, Meyer S. DNA supercoiling: an ancestral regulator of gene expression in pathogenic bacteria? Comput Struct Biotechnol J. 2019;17:1047-55.

38. Dorman CJ. DNA supercoiling and transcription in bacteria: a two-way street. BMC Mol Cell Biol. 2019;20:26.

39. Dorman CJ, Dorman MJ. DNA supercoiling is a fundamental regulatory principle in the control of bacterial gene expression. Biophys Rev. 2016;8: 209-20.
40. Yildirim A, Feig M. High-resolution 3D models of Caulobacter crescentus chromosome reveal genome structural variability and organization. Nucleic Acids Res. 2018;46:3937-52.

41. Brocken DJW, Tark-Dame M, Dame RT. The organization of bacterial genomes: towards understanding the interplay between structure and function. Curr Opin Syst Biol. 2018;8:137-43.

42. Dillon SC, Dorman CJ. Bacterial nucleoid-associated proteins, nucleoid structure and gene expression. Nat Rev Microbiol. 2010;8:185-95.

43. Sobetzko P, Travers A, Muskhelishvili G. Gene order and chromosome dynamics coordinate spatiotemporal gene expression during the bacterial growth cycle. Proc Natl Acad Sci U S A. 2012;109:E42-50.

44. Dame RT, Tark-Dame M. Bacterial chromatin: converging views at different scales. Curr Opin Cell Biol. 2016:40:60-5.

45. Brandão HB, Paul P, Van Den Berg AA, Rudner DZ, Wang X, Mirny LA. RNA polymerases as moving barriers to condensin loop extrusion. Proc Natl Acad Sci U S A. 2019:116:20489-99.

46. Jin DJ, Cabrera JE. Coupling the distribution of RNA polymerase to global gene regulation and the dynamic structure of the bacterial nucleoid in Escherichia coli. J Struct Biol. 2006;156:284-91.

47. Jin DJ, Mata Martin C, Sun Z, Cagliero C, Zhou YN. Nucleolus-like compartmentalization of the transcription machinery in fast-growing bacterial cells. Crit Rev Biochem Mol Biol. 2017:52:96-106.

48. Yang S, Kim S, Kim DK, Jeon An H, Bae Son J, Hedén Gynnå A, et al. Transcription and translation contribute to gene locus relocation to the nucleoid periphery in E coli. Nat Commun. 2019;10:5131.

49. Weng X, Bohrer CH, Bettridge K, Lagda AC, Cagliero C, Jin DJ, et al. Spatial organization of RNA polymerase and its relationship with transcription in Escherichia coli. Proc Natl Acad Sci U S A. 2019;116:20115-23.

50. Martin CM, Sun Z, Zhou YN, Jin DJ. Extrachromosomal nucleolus-like compartmentalization by a plasmid-borne ribosomal RNA operon and its role in nucleoid compaction. Front Microbiol. 2018;9:1115.

51. Engstrom MD, Pfleger BF. Transcription control engineering and applications in synthetic biology. Synth Syst Biotechnol. 2017;2:176-91.

52. Bremer H, Dennis PP. Modulation of chemical composition and other parameters of the cell at different exponential growth rates. EcoSal Plus. 2008;3.

53. Le TBK, Imakaev MV, Mirny LA, Laub MT. High-resolution mapping of the spatial organization of a bacterial chromosome. Science. 2013;342:731-4.

54. Bartlett TM, Bratton BP, Duvshani A, Zhu J, Shaevitz JW, Gitai Z, et al. A periplasmic polymer curves Vibrio cholerae and promotes pathogenesis. Cell. 2017;168:172-85 e15.

55. O'leary NA, Wright MW, Brister JR, Ciufo S, Haddad D, Mcveigh R, et al. Reference sequence (RefSeq) database at NCBI: current status, taxonomic expansion, and functional annotation. Nucleic Acids Res. 2015:44:D733-45.

56. Aziz RK, Bartels D, Best AA, DeJongh M, Disz T, Edwards RA, et al. The RAST server: rapid annotations using subsystems technology. BMC Genomics. 2008;9:75

57. Luo H, Gao F. DoriC 10.0: an updated database of replication origins in prokaryotic genomes including chromosomes and plasmids. Nucleic Acids Res. 2019:47:D74-7.

58. Andrews S. FastQC. Babraham bioinformatics. 2010. Available from: http:// www.bioinformatics.babraham.ac.uk/projects/fastqc. Last accessed 27 Apr 2020

\section{Publisher's Note}

Springer Nature remains neutral with regard to jurisdictional claims in published maps and institutional affiliations.

Ready to submit your research? Choose BMC and benefit from:

- fast, convenient online submission

- thorough peer review by experienced researchers in your field

- rapid publication on acceptance

- support for research data, including large and complex data types

- gold Open Access which fosters wider collaboration and increased citations

- maximum visibility for your research: over $100 \mathrm{M}$ website views per year

At $\mathrm{BMC}$, research is always in progress.

Learn more biomedcentral.com/submission 